Nervenarzt 2016 $\cdot 87: 88-90$

DOI 10.1007/s00115-015-4458-7

Online publiziert: 22. November 2015

(c) Springer-Verlag Berlin Heidelberg 2015

F. Jacobi ${ }^{1,2} \cdot$ M. Höfler ${ }^{1}$ J. Strehle ${ }^{1} \cdot$ S. Mack ${ }^{1}$ A. Gerschler ${ }^{1}$ L. Scholl .

M.A. Busch ${ }^{3} \cdot$ U. Maske ${ }^{1} \cdot$ U. Hapke ${ }^{3} \cdot$ W. Gaebel ${ }^{4} \cdot$ W. Maier ${ }^{5,6} \cdot$ M. Wagner $^{5,6}$. J. Zielasek ${ }^{4} \cdot$ H.-U. Wittchen ${ }^{1}$

${ }^{1}$ Institut für Klinische Psychologie und Psychotherapie, Center of Epidemiology and Longitudinal, Studies (CELOS), Technische Universität Dresden, Dresden

2 Psychologische Hochschule Berlin, Berlin

${ }^{3}$ Abteilung für Epidemiologie und Gesundheitsmonitoring, Robert Koch-Institut, Berlin

${ }^{4}$ Klinik für Psychiatrie und Psychotherapie, Medizinische Fakultät, Heinrich-Heine-Universität, Düsseldorf

${ }^{5}$ Klinik für Psychiatrie und Psychotherapie, Universität Bonn, Bonn

${ }^{6}$ Deutsches Zentrum für Neurodegenerative Erkrankungen (DZNE), Bonn

\title{
Erratum zu: Psychische Störungen in der Allgemeinbevölkerung. Studie zur Gesundheit Erwachsener in Deutschland und ihr Zusatzmodul „Psychische Gesundheit" (DEGS1-MH)
}

2. Einige Prävalenzen und deren Vertrauensintervalle verändern sich geringfügig. Die Gesamtprävalenz beträgt nun 27,8\% (vormals 27,7\%).

Deutliche Abweichungen finden sich nur bei der Major-Depression mit 6,8\% (statt vormals 6,0\%; Frauen: 9,5 statt 8,4\%, Männer: 4,0 statt 3,4\%).

Da es sich bei DEGS1-MH um Referenzdaten für Deutschland handelt und zukünftig weitere Forschergruppen hiermit arbeiten werden, haben sich die Autoren entschlossen, alle Änderungen, ergänzt um entsprechende weitere methodische Erläuterungen, in einem ergänzenden Artikel zum englischsprachigen Artikel im International Journal of Methods in Psychiatric Research zu publizieren [1].

Die Aussagen, die im Originalbeitrag in Der Nervenarzt getroffen wurden, werden durch die Änderungen an keiner Stelle wesentlich beeinflusst. Lediglich - Tab. 1 „12-Monats-Prävalenzen psychischer Störungen (DSM-IV-TR) in der erwachsenen Allgemeinbevölkerung " muss neu erstellt werden, ansonsten sei auf Jacobi et al. [1] verwiesen. Zitiert wird nach wie vor der Originalbeitrag.

Die Autoren bedauern mögliche Irritationen, die hierdurch entstanden sein mögen.

\section{Korrespondenzadresse}

\section{Prof. Dr. F. Jacobi}

Institut für Klinische Psychologie und Psychotherapie, Center of Epidemiology and Longitudinal, Studies (CELOS), Technische Universität Dresden

Chemnitzer Str. 46, 01187 Dresden

frank.jacobi@tu-dresden.de
Die Online-Version des Originalartikels können Sie unter http://dx.doi.org/10.1007/s00115-0133961-y finden. 
Tab. 1 12-Monats-Prävalenzen psychischer Störungen (DSM-IV-TR) in der erwachsenen Allgemeinbevölkerung ${ }^{a}$

\section{Störung bzw. Störungsgruppe (mit ICD-10-}

Code)

Psychische Störung aufgrund medizinischem

Krankheitsfaktor oder substanzinduzierte Stö-

rung (F06)

\begin{tabular}{l} 
Störung durch Substanzgebrauch (F1) \\
\hline $\begin{array}{l}\text { Störung durch Substanzgebrauch (ohne Nikotin- } \\
\text { abhängigkeit) }\end{array}$
\end{tabular}

12-Monats-Prävalenz: \% (95\%-KI)

\begin{tabular}{llll}
\hline Frauen & Männer & Gesamt & ${\text { In } \text { Millionen }^{\mathbf{b}}}^{1,2(0,8-1,9)}$ \\
$1,2(0,8-1,8)$ & $1,2(0,9-1,6)$ & 0,8
\end{tabular}

$14,0(12,5-15,7)$

$19,4(17,6-21,4)$

$16,7(15,5-18,0)$

10,7

$3,5(2,8-4,5)$

$8,0(6,7-9,4)$

$5,7(5,0-6,6)$

3,7

\begin{tabular}{l} 
- Alkoholmissbrauch \\
\hline - Alkoholabhängigkeit \\
- Medikamentenmissbrauch \\
- Medikamentenabhängigkeit \\
- Nikotinabhängigkeit
\end{tabular}

Mögliche psychotische Störung (F2 und andere

Gruppen psychotischer Störungen) ${ }^{d}$

\begin{tabular}{|c|c|c|c|c|}
\hline Affektive Störungen (F3) & $13,1(11,5-14,8)$ & $6,4(5,4-7,6)$ & $9,8(8,8-10,8)$ & 6,3 \\
\hline - Unipolare Depression & $11,3(9,8-12,9)$ & $5,1(4,3-6,1)$ & $8,2(7,3-9,2)$ & 5,3 \\
\hline - Major-Depression & $9,5(8,2-11,1)$ & $4,0(3,3-4,9)$ & $6,8(6,0-7,7)$ & 4,4 \\
\hline - Dysthyme Störung & $2,1(1,6-2,8)$ & $1,2(0,8-1,8)$ & $1,7(1,3-2,1)$ & 1,1 \\
\hline - Bipolare Störung & $1,7(1,2-2,5)$ & $1,3(0,8-2,0)$ & $1,5(1,1-2,0)$ & 1,0 \\
\hline - Bipolar I & $1,1(0,8-1,7)$ & $0,9(0,5-1,5)$ & $1,0(0,7-1,4)$ & 0,6 \\
\hline - Bipolar II & $0,7(0,4-1,4)$ & $0,5(0,2-1,1)$ & $0,6(0,4-1,0)$ & 0,4 \\
\hline Angststörung (F40, F41) & $21,4(19,5-23,4)$ & $9,3(8,1-10,8)$ & $15,4(14,2-16,6)$ & 9,9 \\
\hline - Panikstörunge & $2,8(2,2-3,6)$ & $1,2(0,8-1,8)$ & $2,0(1,6-2,5)$ & 1,3 \\
\hline - Agoraphobie & $5,6(4,6-6,9)$ & $2,3(1,7-3,1)$ & $4,0(3,4-4,7)$ & 2,6 \\
\hline - Soziale Phobie & $3,6(2,7-4,9)$ & $1,9(1,4-2,6)$ & $2,8(2,2-3,4)$ & 1,8 \\
\hline - Generalisierte Angststörung & $3,0(2,2-4,0)$ & $1,5(1,1-2,2)$ & $2,3(1,8-2,9)$ & 1,5 \\
\hline - Spezifische Phobien ${ }^{f}$ & $15,5(13,9-17,2)$ & $5,1(4,2-6,2)$ & $10,3(9,3-11,4)$ & 6,6 \\
\hline Zwangsstörung & $4,0(3,1-5,1)$ & $3,3(2,5-4,2)$ & $3,6(3,0-4,3)$ & 2,3 \\
\hline Posttraumatische Belastungsstörung & $3,6(2,8-4,7)$ & $0,9(0,6-1,5)$ & $2,3(1,8-2,9)$ & 1,5 \\
\hline Somatoforme Störung $(\mathrm{F} 45)^{g}$ & $5,3(4,3-6,4)$ & $1,7(1,3-2,4)$ & $3,5(3,0-4,2)$ & 2,2 \\
\hline$-\mathrm{SSI} 4,6$ & $0,9(0,6-1,6)$ & $0,6(0,4-1,0)$ & $0,8(0,6-1,1)$ & 0,5 \\
\hline -Schmerzstörung & $5,1(4,1-6,2)$ & $1,3(0,9-1,8)$ & $3,2(2,6-3,8)$ & 2,1 \\
\hline Essstörung (F50) & $1,4(0,9-2,1)$ & $0,5(0,3-0,9)$ & $0,9(0,7-1,3)$ & 0,6 \\
\hline - Anorexia nervosa & $1,2(0,7-1,8)$ & $0,3(0,2-0,8)$ & $0,8(0,5-1,1)$ & 0,5 \\
\hline - Bulimia nervosa & $0,3(0,2-0,5)$ & $0,1(0,0-0,3)$ & $0,2(0,1-0,3)$ & 0,1 \\
\hline - Binge-eating-Störung & $0,1(0,0-0,4)$ & $0,1(0,0-0,2)$ & $0,1(0,0-0,2)$ & 0,1 \\
\hline $\begin{array}{l}\text { Irgendeine der genannten (ohne Nikotinab- } \\
\text { hängigkeit) }\end{array}$ & $33,5(31,4-35,7)$ & $22,1(20,2-24,1)$ & $27,8(26,4-29,3)$ & 17,8 \\
\hline - Unter diesen mit einer Diagnose & 49,8 & 63,2 & 55,0 & 9,8 \\
\hline -Zwei Diagnosen & 22,2 & 21,8 & 22,0 & 3,9 \\
\hline - Drei Diagnosen & 12,0 & 6,9 & 10,0 & 1,8 \\
\hline - Vier und mehr Diagnosen & 16,0 & 8,2 & 13,0 & 2,3 \\
\hline
\end{tabular}

aDEGS1-MH, nach Korrektur gemäß [1]; $n=5303$ (davon imputierte Werte von $n=820$ mit fehlenden Werten in diagnostischen Variablen), Daten (\% und Konfidenzintervalle) gewichtet nach Alter, Geschlecht und Designfaktoren; ohne Anwendung von DSM-IV-Hierarchieregeln; eine Addition der einzelnen Prävalenzen (insgesamt und in Unterkategorien) ergibt aufgrund Komorbidität der Diagnosen untereinander eine höhere Zahl als die jeweils angegebene Gesamtprävalenz und ist daher nicht zulässig. bBezugsgröße: 64,1 Mio. Deutsche im Alter von 18 bis 79 Jahren zum 31.12.2010. Zu beachten ist, dass sich a) die Häufigkeiten in verschiedenen Gruppen (z. B. Geschlecht, Altersgruppen) ungleich verteilen und sich die Angaben in Millionen hier auf den jeweiligen Gesamtdurchschnitt beziehen; b) auch die Angaben in Millionen sollen mit entsprechendem Vertrauensintervall interpretiert werden (vgl. Spalte „Gesamt"). ${ }^{\circ}$ Ohne Missbrauch/Abhängigkeit illegaler Drogen (nicht erhoben). ${ }^{d}$ Screening für Schizophrenie und andere psychotische Störungen ohne weitere Differenzialdiagnose; enthält psychotische Merkmale bei affektiven Störungen sowie psychotische Störungen aufgrund medizinischem Krankheitsfaktor und substanzinduzierte psychische Störungen. 'Mit und ohne Agoraphobie. Tierphobien, Phobien vor Naturereignissen (z. B. Gewitter), situationale Phobien (z. B. Höhe), Blut-/Spritzen-/Verletzungsphobien. ${ }^{9}$ Somatic Symptom Index 4,6 (Escobar et al. [42]), Schmerzstörung. 


\section{Literatur}

1. Jacobi F, Höfler M, Strehle J et al (2015) Twelvemonths prevalence of mental disorders in the German Health Interview and Examination Survey for Adults - Mental Health Module (DEGS1$\mathrm{MH})$ : a methodological addendum and correction. Int J Methods Psychiatr Res. [published online in Wiley Online Library (wileyonlinelibrary.com) doi:10.1002/mpr.1479]

\section{Schlaflosigkeit macht schmerzempfindlicher}

Studien der Donau-Universität Krems zeigt beachtliche Effekte Der Zusammenhang zwischen Schlafqualität und chronischem Schmerz ist in der Forschung seit langem bekannt. Sowohl die Schlafdauer, als auch die Schlafqualität - beispielsweise Schlafunterbrechungen - beeinflussen die Schmerzintensität. Wie Studien zeigen, erhöht eine Schlafdauer unter sechs Stunden die Stärke von Schmerzen am Folgetag. Viele Studien belegen dabei eine Wechselwirkung: Chronische Schmerzen stören den Schlaf, umgekehrt erhöht geringe Schlafqualität die Wahrnehmung von Schmerzen. Unklar war bisher das Ausmaß, in dem Schlafmangel die Schmerzwahrnehmung erhöht. Die nun vorliegende Meta-Analyse, unter der Leitung von Prof. Dr. Pieh, zeigt einen Effekt von $\mathrm{SMD}=0.62$. Dieser Effekt ist durchaus beachtlich. Im Vergleich dazu, die Effekte von Schmerzmittel bei chronischen Schmerzen rangieren von $\mathrm{SMD}=0.41$ für nicht-Opiate (wie z. B. Ibuprofen) bis $S M D=0.46$ für Opiate.

Besserer Schlaf als Beitrag zur Linderung chronischer Schmerzen

Wie die Analyse im Detail zeigt, verändert Schlaflosigkeit sowohl das Schmerzempfinden als auch die Schmerzschwelle. Das Ergebnis legt daher nahe, dass PatientInnen mit chronischen Schmerzen, die auch unter Schlaflosigkeit leiden, von therapeutischen Maßnahmen zur Verbesserung der Schlafqualität profitieren könnten. Laut der Autorlnnen sollten zukünftige Studien die klinische Bedeutung des Ergebnisses klären.

Über die Studie

Für die Untersuchung wurden fünf Studien mit $\mathrm{N}=190$ Probandlnnen zur Analyse zwischen Untersuchungsgruppen (between-group Analyse) und zehn Studien ( $\mathrm{N}=266$ ) für die Analyse innerhalb von Untersuchungsgruppen (within-group Analyse) untersucht. Die in den Studien untersuchten Gruppen wurden komplettem, teilweisen und keinem Schlafentzug ausgesetzt. Ausgangspunkt war eine systematische Literatursuche in den Datenbanken PubMed, Cochrane, Psyndex, Psycinfo und Scopus. Die ausgewählten Studien wurden mit einem Paneldatenmodell (Random Effect Model) analysiert.
Marlene Schrimpf, Gregor Liegl, Markus Boeckle, Anton Leitner, Peter Geisler, Christoph Pieh, The effect of sleep deprivation on pain perception in healthy subjects: a meta-analysis, Sleep Medicine (2015) doi:10.1016/j.sleep.2015.07.022

(10.12.15)

Rückfragen

Prof. Dr. Christoph Pieh

Zentrum für Psychosomatische Medizin und Supervision

Department für Psychotherapie und Biopsychosoziale Gesundheit Donau-Universität Krems

Tel. +43 (0)2732 893-2530 christoph.pieh@donau-uni.ac.at www.donau-uni.ac.at/psymed 